\title{
SCAMP: Assessing body-focused repetitive behaviors
}

\author{
Kenneth W. Phelps, PhD, and Marcus Bethurem, DO
}

$\mathrm{R}$ epetitive behaviors towards the body, such as hair pulling and skin picking, are common. Approximately 5\% of the general population may meet criteria for trichotillomania or excoriation disorder, in which the repetitive behaviors are excessive and impairing. The category of body-focused repetitive behaviors (BFRBs) extends beyond these 2 disorders to include onychophagia (nail biting), onychotillomania (nail picking), and lip or cheek chewing, which in DSM-5 are categorized under Other Specified Obsessive-Compulsive Disorder-BFRB. Of particular concern are trichophagia or dermatophagia, the ritualizing and eating of skin or hair that can lead to gastrointestinal complications. ${ }^{1}$

The prevalence and associated distress from BFRBs have spurred increased research into psychotherapeutic interventions to remediate suffering and curb bodily damage. Under the broader umbrella of behavioral therapy or cognitive-behavioral therapy, the Expert Consensus Treatment Guidelines from the TLC Foundation ${ }^{2}$ describe habit reversal therapy, comprehensive behavioral treatment, and behavioral therapy that is enhanced by acceptance and commitment therapy or dialectical behavioral therapy (DBT) skills. (Although these guidelines also summarize possible pharmacologic interventions, medication for patients with BFRBs is not discussed in this article.)

Understanding the antecedents and consequences of these recurrent behaviors is a key aspect of psychotherapeutic treatments because diverse contingencies reinforce these repetitive behaviors. As with any comprehensive assessment, asking questions to understand the function of the behaviors guides personalized treatment recommendations or referrals. Mansueto et $\mathrm{al}^{3}$ described a systematic approach to assessing BFRBs. Asking questions based on these researchers' SCAMP domains (Sensory, Cognitive, Affective, Motor, Place) can provide patients and clinicians with a clear picture of pulling, picking, or other repetitive behaviors.

Sensory. Start with an assessment of how sensory experiences might play into the cycle. Questions might include: Does the patient see a distinctive hair (eg, color, texture) or skin irregularity that draws them into the behavior? Do they visually inspect the hair or skin before, during, or after? Do they describe a premonitory sensation, such as an itch? Do they have a dermatologic condition that cues interoceptive hypervigilance? Do they taste or smell the scab, excoriate, or hair? Are they particularly attuned to the auditory experiences of the process (ie, hearing the pop or a pull)? Could any substances or medications be impacting the body's restlessness?

Cognitive. Just as we assess common automatic thoughts associated with other psychopathologies, it is important to appre-

\section{Every issue of CURRENT PsYCHIATRY} has its 'Pearls'

\section{Yours could be found here.}

Read the 'Pearls' guidelines for manuscript submission at MDedge.com/ CurrentPsychiatry/page/pearls. Then, share with your peers a 'Pearl' of wisdom from your practice.
Dr. Phelps is Associate Professor of Clinical Psychiatry, and Adjunct Associate Professor of Pediatrics, Prisma Health and the University of South Carolina School of Medicine, Columbia, South Carolina.

Dr. Bethurem is a psychiatrist in Columbia, South Carolina.

\section{Disclosures}

The authors report no financial relationships with any companies whose products are mentioned in this article, or with manufacturers of competing products.

doi: 10.12788/cp.0205 
Understanding the antecedents and consequences of BFRBs is a key aspect of treatment ciate the cognitions that occur during this behavioral chain. Some thoughts involve an intolerance of imperfection: "That hair looks different. I have to remove it." "It is important for pores to be completely clean." Other thoughts may involve granting permission: "I'll just pull one." "It has been a long week so I deserve to do just this one." Certainly, many patients may be thinking about other daily stressors, such as occupational or interpersonal difficulties. Knowing about the patient's mental state throughout the BFRB can guide a clinician to recommend treatment focused on (for example) cognitive-behavioral therapy for perfectionism or approaches to address existing stressors.

Affective. One common assumption is that patients who engage in BFRBs are anxious. While it certainly may be the case, an array of affective states may accompany the repetitive behavior. Patients may describe feeling tense, bored, sad, anxious, excited, relieved, agitated, guilty, worried, or ashamed. It is typically helpful to inquire about affect before, during, and after. Knowing the emotional experiences during and outside of BFRBs can call attention to possible comorbidities that warrant treatment, such as a mood or anxiety disorder. Additionally, dysregulation in affective states during the BFRB may point to useful adjunctive skills, such as DBT.

Motor. Some patients describe being quite unaware of their BFRB (often called "automatic"), whereas for other patients pulling or picking may be directed and within awareness (often called "focused"). It is common for patients to have both automatic and focused behaviors. Questions to understand the motor experience include: Is the patient operating on autopilot when they are engaged in the behavior? Does the behavior occur more often in certain postures, such as when they are seated or lying in bed? Understanding the choreography of the BFRB can help in determining physical barriers to protect the skin or hair.

Place. Finally, ask the patient if they believe certain locations increase the occurrence of the BFRB. For instance, some patients may notice the behavior is more likely to occur in the bathroom or bedroom. Bathrooms often contain implements associated with these behaviors, including mirrors, tweezers, or bright lights. Knowing where the BFRB is most likely to occur can help the clinician develop planning strategies to minimize behavioral engagement. An example is a patient who is more likely to pull or pick on a long commute from work. Planning to have a hat and sweater in their vehicle for the drive home may serve as a deterrent and break the cycle. When considering the place, it may also be helpful to ask about the time of day and presence of others.

Gathering information from the SCAMP domains can lead to individualized approaches to care. Of course, nonsuicidal self-injury, delusional parasitosis, or body dysmorphic disorder are a few of the many differential diagnoses that should be considered during the assessment. After a detailed assessment, clinicians can proceed by collaboratively developing strategies with the patient, referring them to a clinician who specializes in treating BFRBs using a resource such as the TLC Foundation's Find a Therapist directory (https://www.bfrb. org/find-help-support/find-a-therapist), or recommending a self-guided resource such as StopPulling.com or StopPicking.com.

\section{References}

1. Diagnostic and Statistical Manual of Mental Disorders. 5th ed. American Psychiatric Association; 2013.

2. The TLC Foundation for Body-Focused Repetitive Behaviors (2016). Expert consensus treatment guidelines. Accessed November 30, 2021. https://www.bfrb.org/ storage/documents /Expert_Consensus_Treatment Guidelines_2016w.pdf

3. Mansueto CS, Vavricheck SM, Golomb RG. Overcoming Body-Focused Repetitive Behaviors: A Comprehensive Behavioral Treatment for Hair Pulling and Skin Picking. New Harbinger Publications; 2019. 